\title{
Digitale Praktiken und das Studium
}

Dr. Isabel Steinhardt

\section{Einleitung ${ }^{1}$}

Digitale Technologien sind allgegenwärtig - auch in der Hochschule. Mit dem Web 2.0 sind pädagogische Hoffnungen verbunden, konstruktivistische Lernkonzeptionen durch informelles, individuelles, selbstgesteuertes, kooperatives, mobiles und gemeinschaftliches Lernen zu verwirklichen (Meister/Meise 2010, S. 183). Es wird nicht mehr in Frage gestellt, dass an Hochschulen die Nutzung digitaler Technologien für Studium und Lehre von hoher Bedeutung ist und ausgebaut werden sollte, sowohl unter strategischen als auch inhaltlichen Aspekten (Wannemacher 2016). Entsprechend wird die Digitalisierung in Studium und Lehre durch diverse Förderlinien sowie politische Forderungen des Bundes und der Länder vorangetrieben (Getto/Hintze/Kerres 2018). In Frage gestellt wird allerdings, inwiefern digitale Technologien dabei zu mehr Bildungsteilhabe und zu einem Abbau sozialer Ungleichheiten führen (ebd.). Markus Deimann (2020, S. 6) führt aus, dass in Bezug auf digitale Lehre ein „instrumentelles Verständnis von Bildungstechnologien, d.h. der Glaube, damit “irgendwie” Lernen und Lehren zu verbessern“, herrsche. Durch dieses instrumentelle Verständnis wird selten diskutiert, dass Technologien nicht neutral sind, sondern „Einfluss auf die pädagogische Praxis“ (Deimann 2020, S. 12) nehmen und dadurch die Praktiken der Beteiligten beeinflussen.

Welche Wechselwirkung zwischen Bildungsbeteiligten und digitalen Technologien existieren und welche Praktiken durch diese Wechselwirkung entstehen, ist in Bezug auf Studierende wenig erforscht (Pensel/Hofhues 2018; Steffens/Schmitt/Aßmann 2018). Eine kritische Diskussion der Praktiken in Bezug auf digitale Technologien von Studierenden im Studium ist aus einer Ungleichheitsperspektive von Bedeutung. Denn im Gegensatz zu gängigen Mythen gibt es keine „Digital Natives“ oder eine „Netzgeneration“ (Bond et al. 2018). Studierende verfügen nicht automatisch über die Fähigkeiten, kompetent mit digitalen Technologien und dem Web 2.0 umzugehen. Wie Malte Persike und Julius-David Friedrich (2016) aufzeigen wird die private Nutzung digitaler Medien nicht einfach in Lernkontexte übertragen. Vielmehr zeigt die Forschung zur digitalen Wissenskluft (Zillien 2013), dass die Praktiken von dem zur Verfügung

\footnotetext{
${ }^{1}$ Ich danke Sinen Ben Mekki, Leon Pomnitz und Claudia Weber für das Erheben, Transkribieren der Interviews und erste analytische Überlegungen, die ich in diesem Artikel verwendet habe. Mein Dank gilt auch Dr. Christiane Rittgerott und Sinen Ben Mekki für viele hilfreiche Anregungen zu diesem Beitrag. Zudem möchte ich mich beim Verlag Beltz Juventa bedanken, der mir ermöglicht hat diesen Beitrag vorab als Preprint zu veröffentlichen.
} 
stehenden kulturellen, ökonomischen, symbolischen und sozialen Kapital (Bourdieu 1987a; 1987b) abhängig sind. Forschung aus den USA mit Jugendlichen zeigt zudem, dass sich die Nutzungspraktiken digitaler Technologien je nach Habitus unterscheiden und unterschiedliche Inkorporationen digitaler Technologien stattfinden (Ignatow/Robinson 2017; Robinson et al. 2015). Die Frage nach der Aneignung und Inkorporation digitaler Technologien wird auch in den Science and Technology Studies u.a. anhand des Phänomens digitaler Praktiken untersucht (Allert et al. 2018; Carstensen 2016; 2017; Fries 2019; Leger/Panzitta/Tiede 2018; Zillien/Fröhlich/Kofahl 2016). Das Spezifische bei digitalen Praktiken besteht darin, dass der Umgang mit digitale Technologien inkorporiert und damit ein Leben ohne nicht mehr vorstellbar ist (Carstensen 2016). Für das Studium würde das bedeuten, dass sich durch digitale Technologien neue Praktiken herausbilden, die es zuvor nicht gegeben hat und die ohne digitale Technologien nicht hervorgebracht worden wären. Nach Dorothee M. Meister und Bianca Meise (2010) könnten sich z.B. durch Social Media informelle selbstgesteuerte, kooperative, mobile und gemeinschaftliche Praktiken des Lernens entwickeln, die ohne digitale Technologien vorher nicht möglich waren.

Die Forschungsstränge zu digitalen Praktiken und sozialer Ungleichheit kombinierend wird in dieser explorativen Studie gefragt: Welche digitalen Technologien werden im Studium genutzt und findet eine Inkorporation (Bourdieu 1987a) digitaler Technologien für das Studium statt, welche zum Entstehen digitaler Praktiken führen? Daran anknüpfend wird gefragt, ob sich Unterschiede in der Aneignung digitaler Technologien, d. h. Unterschiede von digitalen Praktiken bei Studierenden zeigen und ob dadurch neue Ungleichheiten entstehen oder soziale Ungleichheiten gefestigt werden?

Um einen ersten Einblick in Praktiken in Bezug auf digitale Technologien zu erhalten, wurden narrative Interviews (Schütze 1977) mit Studierenden geführt, die habitushermeneutisch (Bremer/Teiwes-Kügler 2013) ausgewertet wurden. Das Sample und das Vorgehen werden im Abschnitt „Methode und Sample“ beschrieben, bevor die Praktiken der Studierenden aufgezeigt und Gemeinsamkeiten und Unterschiede herausgearbeitet werden. In einem Fazit werden die Praktiken der Studierenden eingeordnet und Implikationen für die Digitalisierung an Hochschulen benannt. Zunächst wird kurz der aktuelle Forschungsstand zu digitalen Praktiken dargestellt. 


\section{Digitale Praktiken}

Die Frage, wie Subjekte mit digitalen Technologien umgehen und eine Inkorporation stattfindet wird vermehrt diskutiert (Allert/Asmussen/ Richter 2018; Carstensen 2017; Friese 2019; Houben/Prietl 2018; Schmid/Veits/Vorrath 2018). Digitale Praktiken werden zunächst als soziale Praktiken gefasst. Der Begriff der Praktiken wird dabei vor allem in der Praxistheorie und den Science and Technology Studies diskutiert (Carstensen 2017; Schäfer 2016b). Pierre Bourdieu (1987b, S. 101) definiert Praktiken wie folgt:

„Als Produkt der Geschichte produziert der Habitus individuelle und kollektive Praktiken, also Geschichte, nach den von der Geschichte erzeugten Schemata; er gewährleistet die aktive Präsenz früherer Erfahrungen, die sich in jedem Organismus in Gestalt von Wahrnehmungs-, Denk- und Handlungsschemata niederschlagen und die Übereinstimmung und Konstantheit der Praktiken im Zeitverlauf viel sicherer als alle formalen Regeln und expliziten Normen zu gewährleisten suchen.“

Praktiken fußen auf den inkorporierten Wahrnehmungs-, Denk- und Handlungsschemata (die im Habitus zum Ausdruck kommen), die unter bestimmten räumlichen und zeitlichen Umständen entwickelt werden (Bourdieu 1987b). Die entwickelten Wahrnehmungs-, Denk- und Handlungsschemata unterscheiden sich aufgrund der sozialen Stellung des Individuums, die wiederum abhängig von der jeweiligen Kapitalausstattung ist - also vom kulturellen, ökonomischen, sozialen und symbolischen Kapital (Vester 2013, S. 142). Praktiken ergeben sich einerseits in Abhängigkeit von Handlungsmöglichkeiten, die von Habitus und den jeweiligen Kapitalausstattungen beeinflusst sind, und anderseits innerhalb bestimmter sozialer Strukturen (Lenger/Schneickert/Schumacher 2013). Praktiken sind entsprechend erstens von den Umständen, zweitens von den Orten und drittens von den materiellen Rahmungen abhängig (Schmidt 2012). Betrachtet man zudem die Aneignungsprozesse informellen Wissens, wie Bourdieu (1987b) dies in seinem Habitus-Konzept getan hat, müssen die Peergroup sowie Familie und Netzwerke in den Blick genommen werden. Dabei wird das Individuum ,jeweils nur in die Kultur eines bestimmten sozialen Segments“ (Stecher 2005, S. 378) sozialisiert. Entsprechend können Praktiken als Bindeglied zwischen persönlicher Lebensweise und sozialen Strukturen angesehen werden (Zillien/Marr 2013).

Digitale Praktiken sind zudem hochgradig mit materiellen digitalen Artefakten verwoben, da der Umgang mit digitalen Technologien inkorporiert und damit ein Leben ohne nicht mehr vorstellbar wird (Carstensen 2016). Zu nennen sind hier beispielsweise Praktiken der Selbstvermessung durch Fitnessapps, wobei die Selbstvermessung mit anderen via Internet geteilt wird 
(Leger/Panzitta/Tiede 2018) oder Diet-Tracking (Zillien/Fröhlich/Kofahl 2016), Praktiken der Subjektivierung in sozialen Medien (Allert et al. 2018) oder digitale Praktiken in der Arbeit (Carstensen 2017). Die digitalen Praktiken entstehen dabei im Zusammenspiel mit Software, Algorithmen, Metrisierung sowie der dauerhaften Speicherung digitaler persönlicher Daten (Fries 2019).

Im Gegensatz zu diesen Beispielen digitaler Praktiken kann aufgrund der historischen Betrachtung von eLearning, Moocs und anderen digitalen Formaten (Deinmann 2020), davon ausgegangen werden, dass die Aneignung digitaler Technologien für das Studium widerständiger abläuft. Die Anwendung digitaler Technologien in der Lehre ist an deutschen Hochschulen meist beschränkt, z.B. auf die Nutzung von Lernplattformen wie moodle oder stud-IP. Diese Plattformen ersetzen im Grunde die Funktion der Seminarreader, indem Texte hoch- und heruntergeladen werden können, anstatt kopiert zu werden. Funktionen wie Chats, Foren oder Wikis werden eher selten verwendet (Persike/Friedrich 2016). Das heißt, von Lehrenden werden digitale Technologien als Analogon zu analogen Technologien verwendet. Analoge Praktiken werden in den digitalen Raum übertragen, die neuen Praktiken fußen auf alten Praktiken (Schäfer 2016a), das heißt, bewährte Praktiken werden durch digitale Technologien unterstützt. Inwiefern dadurch auch digitale Praktiken entstehen, also Praktiken bei denen digitale Technologien so inkorporiert sind, dass ohne die jeweilige Technologie die Praktik nicht mehr ausführbar ist, gilt es zu untersuchen. Da Praktiken auf die Wiederholung und Nachahmung angewiesen sind und sowohl implizite als auch explizite Elemente enthalten (Bourdieu 1976, S. 192), müssten digitale Praktiken vorgelebt werden, damit Studierende digitale Praktiken in Hochschulen inkorporieren. Es könnte aber auch angenommen werden, dass Studierende digitale Praktiken in Hochschulen mitbringen, die in anderen Zusammenhängen erworben wurden (z.B. Elternhaus, Schule, Peer Group, Arbeitserfahrungen) und auf den Kontext Hochschule übertragen, was Dozierende zwingen könnte, ihre eigenen Praktiken zu verändern.

\section{Methode und Sample}

Praktiken werden in der Praxistheorie klassischerweise durch ethnographisch-beobachtende Methoden ermittelt (Schmidt 2012), können aber auch durch Interviews herausgearbeitet werden, gerade dann, wenn der Zugang zu den Praktiken erschwert ist (Maiwald 2003). Der erschwerte Zugang der Beobachtung ist in Bezug auf digitale Praktiken gegeben, da digitale Technologien überall und jederzeit genutzt werden können. Die Praktiken sind unabhängig von bestimmten Orten und in den digitalen Raum verlagert (Leger et al. 2018). Über narrative In- 
terviews können die Nutzungsgewohnheiten und die genutzten digitalen Technologien aber erzählt und erfragt werden. Deshalb wurde für die vorliegende explorative Studie ein für die Habitushermeneutik (Bremer/Teiwes-Kügler 2013; Lange-Vester/Teiwes-Kügler 2013; TeiwesKügler/Lange-Vester 2018) klassisches Vorgehen gewählt und narrative Interviews (Schütze 1977) mit Studierenden durchgeführt, die einen Fokus auf die Nutzung digitaler Technologien in der Lebensgeschichte legten. Die Habitushermeneutik zielt darauf ab, den Habitus und die damit verbundenen Praktiken von Individuen durch die regelbasierte Interpretation zu rekonstruieren. Im vorliegenden Fall können so die digitalen Praktiken der Studierenden identifiziert und mit den zugrundeliegenden Wahrnehmungs-, Denk- und Handlungsschemata in Verbindung gebracht werden ${ }^{2}$. Dazu wurden die Interviews in einer Auswertungsgruppe mit Studierenden und Promovierenden und in einer kollaborativen Online-Gruppe analysiert (Steinhardt 2018; 2020; Steinhardt/Ikiz-Akınc1 2020).

Um eine kontrastierende und tiefergehende Darstellung zu ermöglichen, wurde aus einem Sample von neun Interviews aus vier Fächern (Geschichte, Jura, Soziale Arbeit und Soziologie) sechs Studierende aus zwei Disziplinen gewählt. Von den sechs Interviews wurden drei von mir durchgeführt und drei durch Studierende eines von mir veranstalteten Lehr-Forschungsprojektes. Alle Interviews wurden aufgezeichnet und transkribiert. Gewählt wurden die Disziplinen Jura und Soziale Arbeit. Die Auswahl dieser Disziplinen erfolgte aufgrund von zwei Faktoren: Zum einen handelt es sich bei beiden Disziplinen um Buchwissenschaften, die einen ähnlichen Diskussionsstand in Bezug auf die Digitalisierung der Lehre aufweisen. Diskutiert wird, dass eine Nähe zum Klienten hergestellt werden muss, weshalb Digitalisierung auch als Thematik aufgegriffen werden sollte (Dauner-Lieb/Kaulbach 2018; Helbig 2017)

Zum anderen aufgrund des unterschiedlichen Prestiges der Disziplin und ihrer daraus resultierenden unterschiedlichen Attraktivität für potentielle Studierende (Becker/Haunberger/Schubert 2010; Schmitt 2010). Zudem war es das Ziel der Untersuchung, ein möglichst breites Spektrum an Studierenden aus unterschiedlichen sozialen Milieus zu erhalten, um möglichst unterschiedliche Praktiken identifizieren zu können. Deshalb wurden Jura und Soziale Arbeit als Gegensatzpaar gewählt. So werden juristische Studiengänge eher von Abiturient*innen aus höheren sozialen Milieus gewählt (Becker et al. 2010). Zudem herrscht eine hohe Berufsvererbung, d.h., der Anteil an Studierenden deren Vater oder Mutter ebenfalls Jurist bzw. Juristin sind, ist im Vergleich, zusammen mit der Medizin, am höchsten (23\%) (Spangenberg/Beuße/Heine 2011, S. 89). Studiengänge der Sozialen Arbeit gelten als prestigearm und

\footnotetext{
${ }^{2}$ Auf die Darstellung der einzelnen Auswertungsschritte musste hier leider aus Platzgründen verzichtet werden.
} 
damit als Aufsteigerfächer für Personen aus sozial schwächeren Milieus (Becker et al. 2010). Zudem gibt es die Möglichkeit, Studienfächer der Sozialen Arbeit auch mit Fachabitur bzw. nach Absolvieren einer Erzieherausbildung zu studieren. In diese Überlegungen eingebunden ist die Erkenntnis von Bourdieu und Passeron (1971), dass es eine Nähe spezifischer Herkunftsmilieus und Habitusformen zu der Kultur des Bildungssystems gibt: „Die privilegiertesten Studenten verdanken ihrem Herkunftsmilieu nicht nur Gewohnheiten, Fähigkeiten und Einstellungen, die für das Studium unmittelbar nützlich sind; sie haben auch andere Kenntnisse, Verhaltensweisen, Interessen und einen ,guten Geschmack` ererbt, die dem Studium indirekt zugutekommen (sic!)“ (Bourdieu/Passeron 1971). Eine Gegenüberstellung dieser Studiengänge, so die Überlegung, erlaubt die Rekonstruktion von möglichst diversen Praktiken, die sich auf unterschiedliche Habitus und damit kontrastierende Wahrnehmungs-, Denk- und Handlungsschemata zurückführen lassen.

\section{Digitale Technologien und Praktiken im Studium}

\section{Praxis A: Nutzung digitaler Technologien aufgrund externen Gegebenheiten}

Patrik ist ein älterer Studierender der Sozialen Arbeit, der bereits zwei Ausbildungen vor seinem Studium abgeschlossen hat und mehrere Jahre berufstätig war. Auch Jasmin hat vor ihrem Bachelorstudium Wirtschaftsrecht eine Ausbildung absolviert. Die Wahl der Ausbildung/en war bei beiden pragmatisch und durch soziale Netzwerke bestimmt, die bei der Vermittlung halfen. Beiden ist gemeinsam, dass sie sich nicht vorstellen konnten, weiter in dem erlernten Beruf tätig zu sein, weshalb sie ein Studium begonnen haben, um sich neu auszurichten. Die Eltern von Patrick und Jasmin haben Berufsausbildungen absolviert und sich aktiv durch Weiterbildungen an die Gegebenheiten des Arbeitsmarktes angepasst. Beide Familien waren nicht bildungsaffin im Sinne einer strukturellen Unterstützung während der Schulzeit. Bei beiden liegt die Orientierung im Leben auf der Familie und den Freunden, wobei das Studium bei Jasmin dazu dient, eine gute Berufsperspektive zu bekommen, um ein abgesichertes Leben zu haben, wohingegen es bei Patrick darum geht, sich selbst weiterzuentwickeln. Er hat bewusst die Sicherheit einer Festanstellung in seinem erlernten Beruf aufgegeben, um das Studium aufzunehmen.

Das Studium nehmen beide als sehr voraussetzungsvoll wahr und sie kämpfen mit den an sie gestellten Anforderungen. Zwar kennen sie relevante Recherchemöglichkeiten, haben aber Schwierigkeiten, die relevanten und einschlägigen Quellen zu identifizieren. Beide berichten von Problemen beim Verfassen von Hausarbeiten und dem Gefühl der Hilflosigkeit. Patrick 
erläutert im Interview zudem ausführlich, welche Schwierigkeiten er damit hat, das Lesepensum der Fachliteratur zu bewältigen und dass er noch damit beschäftigt ist, die für ihn sinnvolle Lesestrategie (Querlesen und Behalten der Inhalte) zu finden.

Sowohl Jasmin als auch Patrick bezeichnen sich selbst als nicht digital affin. Jasmin beschreibt ihren Zugang zu digitalen Medien als „unumgängliche“ Anforderung von außen: „also ich gehör jetzt nich zu denjenigen, die, ähm, gerne oder vo-, vorher gerne so mit Medien, digitalen Medien gearbeitet hat. Das kam dann auch eher so mit der Ausbildung, und auch vor allem mit dem Studieneinstieg dann nach der Ausbildung, ja.“ (Jasmin: Z. 22-26) Auch Patrick berichtet, dass er in seiner Ausbildung mit dem Computer gearbeitet, recherchiert und spezielle Programme beherrscht hat. Beide berichten dies in Nebensätzen und nicht als eine Kompetenzaneignung, die stattgefunden hat. Bei beiden sind es Kompetenzen, die sich in ihren Augen nicht auf das akademische Feld ihres Studiums übertragen lassen und die deshalb keine Bedeutung mehr haben. Insgesamt geben sie digitalen Technologien und der Kompetenz damit umzugehen wenig Relevanz für ihr Leben (im Gegensatz zu Martin und Felix im nächsten Abschnitt). Jasmin ist aufgrund ihres Alters im Gegensatz zu Patrick mit sozialen Medien wie Schüler-VZ, facebook und whatsapp sozialisiert worden. Sie nutzt diese Dienste zur Kommunikation allerdings vor allem, weil sie sonst aus sozialen Zusammenhängen ihrer Peergroup ausgeschlossen wäre.

In Bezug auf das Studium werden digitale Technologien zum einen auf die Recherche im Internet (Texte bzw. Urteile) und die Nutzung des Computers als Schreibgerät reduziert. Beide fühlen sich mittlerweile im Umgang mit digitalen Datenbanken der Universität sicher, die eigentliche Lesearbeit und Kommentierung der Texte bzw. Urteile erfolgt analog auf Papier. Patrick berichtet von einer Recherchepraktik, bei der er beim Abholen des Buches im Bibliotheksregal auch die benachbarten Bücher sichtet, ob dort etwas für ihn Relevantes dabei ist - diese Praktik wurde ihm von einem Freund empfohlen. Es macht den Eindruck, als würde er seinen digitalen Recherchefertigkeiten nicht vertrauen und deshalb zusätzlich analoge Suchbewegungen machen.

Beide erzählen von digitalen Versuchen durch Dozierende in ihrem Studium wie der Nutzung einer Abstimmungs-App (Jasmin) oder als Studienleistung einen Blogbeitrag schreiben zu lassen (Patrick). Die Anforderung, einen Blogbeitrag zu schreiben, ist ungewohnt für Patrick, da ihm das Veröffentlichen und Teilen selbstgeschriebener Texte fremd ist. Die digitalen Praktiken des Web 2.0 verunsichern ihn und er lehnt es ab, sich darauf einzulassen. Auch Jasmin kann keinen Sinn in der Anwendung der Abstimmungs-App ihres Dozierenden erkennen. 
Beiden ist klar, dass digitale Angebote wie Lehrveranstaltungsaufzeichnungen oder die Nutzung von Literaturverwaltungsprogrammen für das Studium sinnvoll sein könnten, sie nutzen diese Angebote aber nicht, da die Beschäftigung mit diesen, für sie neuen, Angeboten „im Moment“ zu zeitintensiv und aufwendig ist. Beide kämpfen mit den analogen Anforderungen des Studiums und sehen zusätzliche digitale Anforderungen als zusätzliche Belastung. Insgesamt zeigt sich, dass Patrick und Jasmin den Umgang mit digitalen Technologien zwar erlernt haben und Kompetenzen im Umgang mit diesen besitzen, allerdings wird diesen Kompetenzen keine Bedeutung beigemessen, weshalb sie auch für das Studium nicht relevant sind.

\section{Praxis B: Digitale Technologien als Unterstützung im (studentischen) Alltag}

Für Martin und Felix steht die sinnvolle Nutzung von digitalen Technologien im Vordergrund. Ihnen geht es nicht um Unterhaltung durch oder die reine Kommunikation über digitale Technologien, wie es für die Praxis der Unterhaltung (s.u.) dargestellt wird. Vielmehr geht es darum, wie Apps, Programme und Technologien sinnvoll in den Lern- und Arbeitsalltag integriert werden können - oder wo für sie die Grenzen liegen. Diese Grundhaltung in Bezug auf digitale Technologien lässt sich allerdings nicht auf ein geteiltes Milieu zurückführen, weshalb davon ausgegangen werden kann, dass die Praxis der sinnvollen Nutzung in verschiedenen Milieus eine Rolle zu spielen scheint.

Bei Martin, der Mitte dreißig ist und bereits Familie hat, waren Medienkompetenzen entscheidend in seinem Leben: So hat er nach dem Fachabitur zunächst ein Studium der Wirtschaftswissenschaften begonnen und nebenbei gearbeitet. Die Arbeitsstelle hat er durch seinen Vater vermittelt bekommen, der in der gleichen Firma gearbeitet hat. Beide Eltern habe eine Berufsausbildung, der Vater hat sich in der Firma hochgearbeitet, die Mutter arbeitet bei einer Stadtbehörde und ist verbeamtet. Martin betont, dass er seine Stelle vor allem aufgrund seiner ExcelProgrammier-Kompetenzen erhalten hat, die er sich selbstständig während seiner Schulzeit spielerisch angeeignet hatte. Aufgrund familiärer Verpflichtungen hat er Vollzeit gearbeitet und das Wirtschaftswissenschaftenstudium nicht abgeschlossen. Da seine Frau nun nach ihrem Studium eine feste Stelle hat, hat er beschlossen, ein Studium der Sozialen Arbeit zu beginnen, da er in diesem Bereich eine Chance der Selbstverwirklichung sieht.

Auch Felix ist versiert im Umgang mit digitalen Technologien. Seit seiner Kindheit hat er den Umgang mit digitalen Technologien spielerisch erlernen können und wurde mit den neuesten Geräten ausgestattet, was auf ein ökonomisch gut ausgestattetes Elternhaus schließen lässt. Sein Vater ist Akademiker und hat eine gehobene Managementfunktion. Die Mutter hat eine abgeschlossene Berufsausbildung und ist als Bürofachkraft tätig. Felix besitzt nur Apple-Geräte und 
benennt diese distinguierend dauerhaft im Interview. Zunächst hatte er Jura als Nebenfach zur Politikwissenschaft gewählt, dann aber zu Jura gewechselt, da man damit „tatsächlich was anfangen kann“.

Beide können, im Gegensatz zu Patrick und Jasmin, mit den Anforderungen des Studiums gut umgehen. Felix benennt implizite Regeln seines Studienfaches, indem er erzählt, dass zwar alle Beck-Online (eine juristische Fachdatenbank) nutzen, um Gesetzeskommentare u.Ä. zu lesen, „[i]nteressanterweise wird es aber schon natürlich immer so zitiert, als ob man es analog gelesen hätte“ (Felix: Z. 30). Da Felix eine breite Auswahl an technischen Geräten hat, überlegt er sich im Vorfeld, welches Gerät er nutzen möchte, abhängig beispielsweise von der Größe des Bildschirms, um am besten lesen zu können. Beide reflektieren ihren Umgang mit digitalen Angeboten und haben Strategien entwickelt, diese zu kontrollieren. So hat Felix keine facebook-App auf dem iPhone, um den Konsum zu regulieren. Martin dagegen liest am liebsten Texte auf Papier, um nicht durch das Internet abgelenkt zu werden und ins „ziellos Surfen“ abzugleiten. Beide nutzen facebook vor allem, um mit Personen im Ausland Kontakt zu halten, Martin ist zudem in einer facebook-Ersti-Gruppe, um wichtige Informationen über seinen Studiengang zu erhalten und sich zu vernetzen. Felix hat auch eine whatsapp-Mensagruppe, Martin wiederum lehnt whatsapp ab, da er lieber direkten Kontakt (per Telefonanruf) zu den Personen hält. Von digitalen Angeboten oder Seminaren, in denen digitale Technologien explizit genutzt wurden, berichten beide nicht. Martin kennt moodle und nutzt die Plattform auch zum Downloaden von Texten. Andere Angebote, die von Dozierenden oder der Universität kommen könnten, spielen in seinem Studium aber keine Rolle.

\section{Praxis C: Social Media zur Unterhaltung - aber Ablehnung für das Studium}

Im Gegensatz zu Felix ist es Laura und Annika nicht wichtig, die neuesten oder teuersten Geräte zu besitzen, denn für sie dient der Computer vornehmlich als Schreibgerät. Beide sind nicht bereit, für technische Geräte (ausgenommen das Smartphone) viel Geld auszugeben. Das Smartphone ist zentraler täglicher Begleiter, weil darüber sämtliche Kommunikation und Unterhaltung abgewickelt wird. Beide sind mit Social Media in ihrer Adoleszenz sozialisiert und sind früh z.B. bei Studi-VZ und dann bei facebook eingestiegen. Die sozialen Medien sind für beide wichtiger Bezugspunkt, um sich in der Jugend auszuprobieren: „Es war auch irgendwie so ' $\mathrm{n}$ bisschen Selbstdarstellung und man konnte andere halt so 'n bisschen (1) halt quasi so stalken, also die so so beobachten“ (Annika: Z: 55-56). Für beide ist es aber wichtig, die Hoheit über ihre Identität zu wahren, weshalb sie kaum Bilder von sich posten und sich selbst als User, aber nicht als Producer wahrnehmen. 
Sowohl Annika als auch Laura kommen aus akademischen Elternhäusern, in denen beide Elternteile studiert haben und Bildungsaufsteiger*innen sind. Lauras Mutter arbeitet allerdings nicht mehr in einem akademischen Beruf, sondern war gezwungen umzuschulen. Bei Beiden sind die Väter technisch affin, wohingegen die Mütter technischen Neuerungen eher ablehnend gegenüberstehen. Als Ausdruck dieses Widerstreits in der Familie haben beide erst spät in ihrer Schulzeit ein Smartphone erhalten, was für Laura unproblematisch war, bei Annika aber zu Ausgrenzungsgefühlen geführt hat. Sie hatte das Problem, dadurch nicht mit ihrer Peergroup per whatsapp kommunizieren zu können und fühlte sich abgehängt. Social media zu nutzen ist für beide ein zentrales Moment, um zu der jeweiligen Peergroup Anschluss zu halten und dazu zu gehören. Auch der Studienalltag wird per whatsapp koordiniert und die Freizeitgestaltung wird von facebook-Ankündigungen bestimmt.

Laura und Annika studieren beide einen juristischen Studiengang. Sie haben im Studium keine größeren Probleme. Durch Praktika orientieren sie sich bereits auf dem Arbeitsmarkt und eruieren früh im Studium mögliche berufliche Perspektiven. Dabei erkunden sie unterschiedliche Berufe und sind in Hinblick auf die spätere Berufswahl offen. Digitale Technologien spielen in ihrem Studium kaum eine Rolle. Ähnlich wie Jasmin haben auch sie Erfahrung mit UmfrageApps gemacht, finden diese aber im Studium unangemessen. Laura hatte zudem ein Seminar, indem sie via Internetplattform kooperativ an einem juristischen Fall arbeiten musste. Dies erledigt sie, allerdings ohne großen Enthusiasmus und ist froh, dass es eine einmalige Veranstaltung war. Für Annika hat YouTube eine große Relevanz in ihrem Leben, da sie sich gerne darüber unterhalten lässt. In der Schulzeit und zu Beginn ihres Studiums hat sie YouTube auch für Lernzwecke genutzt, lehnt dies aber mittlerweile als zu wenig wissenschaftlich/akademisch für ihren Studiengang ab. Annika berichtet von der Nutzung von googledocs und Skype während eines Praktikums und reflektiert, dass dadurch einerseits eine einfache örtlich ungebundene Arbeitsweise möglich ist, andererseits eine Entgrenzung von Arbeit stattfinden kann. Sie nimmt damit sowohl die positiven als auch negativen Seiten digitaler Technologien wahr.

\section{Fazit}

Die hier vorliegende explorative Studie in den Fächern Jura und Soziale Arbeit hat für die sechs Fälle aufgezeigt, dass keine digitalen Praktiken im Studium zu finden sind. Zwar haben die Studierenden digitale Praktiken v. a. in Bezug auf Kommunikation und Social Media, diese werden aber nicht für das Studium genutzt. Die interviewten Studierenden nutzen alle digitale Technologien, allerdings mit unterschiedlicher Intensität. Insgesamt beschreiben die Studieren- 
den dabei grundlegende Nutzungen wie z. B. das Schreiben auf dem Computer oder das Recherchieren im Internet. Hier zeigen sich einige Praktiken, die durch digitale Technologien erleichtert werden, wie das Suchen nach Büchern online statt im alten Zettelkatalog. Das Beispiel beschreibt eine Übertragung einer vorher analogen Praktik in den digitalen Raum, aber keine digitale Praktik fürs Studium. Die Nutzung von aufgezeichneten Vorlesungen, Umfrage-Apps oder das Anregen von kooperativem Arbeiten im Studium wird als Seltenheit berichtet und als wenig gewinnbringend für den eigenen Lernzugewinn abgelehnt.

Digitale Praktiken der Kommunikation mittels Smartphone und z. B. Whatsapp werden zur Kommunikation mit Kommiliton*innen verwendet, nicht aber zur Kommunikation mit den Dozierenden. Wohingegen die Kommunikation via E-Mail, die neben dem persönlichen Kontakt im Klassenraum als zentrales Medium angesehen werden kann, in den Interviews nicht erwähnt wird. Hier könnte vermutet werden, dass die E-Mail-Kommunikation schon so inkorporiert ist, dass es keiner Erwähnung mehr bedarf. Die doch meist noch förmliche Kommunikation (im Gegensatz zur schnellen Whatsapp-Nachricht) stellt aber erneut die Abbildung einer analogen Praktik (des Briefschreibens) im digitalen Raum dar. Symbolisch verdeutlicht den analog-digitalen Übertrag schon allein das Briefsymbol der E-Mail.

Diese Ergebnisse decken sich mit den Ergebnissen von Persike und Friedrich (2016), die eine Diskrepanz zwischen privater Nutzung von z.B. Social Media und der Nutzung für das Studium festgestellt haben. Es kann angenommen werden, dass die Studierenden bereits inkorporiert haben, dass im akademischen Feld der Hochschule nur kodifiziertes Wissen honoriert wird, informelles Wissen dagegen nicht, weshalb die Nutzung von z. B. YouTube Angeboten für das Studium abgelehnt wird.

Bei der Rekonstruktion der Praktiken zeigen sich Unterschiede zwischen den Studierenden, die darauf hindeuten, dass soziale Ungleichheiten durch die vermehrte Nutzung digitaler Technologien gefestigt werden könnten. Die Digitalisierung des Studiums könnte also, wie in der digitalen Wissenskluft (Zillien 2013) bereits für die Gesellschaft beschrieben, zu einer Ungleichheitsverschärfung führen. In den vorliegenden Beispielen hat eine umfangreiche Digitalisierung des Studiums nicht stattgefunden, weshalb im Folgenden nur Annahmen getroffen werden können, welche Auswirkung digitale Lehre auf die Studierenden hätte.

Wie die Rekonstruktion der Praktiken gezeigt hat, eignen sich Jasmin und Patrick, die aus weniger bildungsnahen Elternhäusern stammen, digitale Technologien nur an, wenn dies von ihnen gefordert wird und benötigen dafür klare Hilfestellungen. Durch die vermehrte Nutzung digitaler Technologien im Studium würden für sie neue Anforderungen erwachsen, während 
sie mit den Anforderungen des „analogen“ Studiums kämpfen. Die eigenen Handlungspraktiken sind nicht darauf ausgerichtet neue digitale Technologien zu entdecken oder intrinsisch motiviert zu erlernen. Ohne Unterstützungsleistung könnte hier entsprechend eine zusätzliche Überforderung stattfinden.

Im Gegensatz zu Jasmin und Patrick haben Martin und Felix keine Schwierigkeiten in ihrem Studium. Für sie stellen digitale Technologien eine Unterstützungsmöglichkeit im Studium dar. Im Studium wird geprüft, welche Technologien oder Apps für welche Zusammenhänge gewinnbringend genutzt werden können und ob es zielführender ist keine digitalen Technologien einzusetzen, um sich z. B. nicht abzulenken. Dabei helfen ihnen ihr kulturelles Kapital und die bereits erworbenen Kompetenzen aus anderen Lebenszusammenhängen. Für beide würde die vermehrte Inklusion digitaler Technologien in das Studium keine Hürde darstellen.

Für Annika und Laura spielen Social Media wie facebook und YouTube eine große Rolle im täglichen Leben für die Kommunikation und v. a. zur Freizeitgestaltung, für das Studium wird die Nutzung von Social Media aber abgelehnt. Ständiger Begleiter ist das Smartphone, das entsprechend teuer sein darf, wohingegen für das Studium nur ein alter Computer als digitale Schreibmaschinen verwendet wird. Annika und Laura haben die ihnen vorgelebten Praktiken im Studium, bei denen digitale Technologien keine Rolle spielen, adaptiert. Wären sie in ein digitalisiertes Studium sozialisiert worden, hätten sie sich aber schnell zurechtgefunden. So zeigen ihre Erfahrungen in Praktika, in denen digitale Technologien eine größere Rolle spielen, dass sie mit der Adaption keine Probleme haben.

Die hier vorgestellte Studie zeigt unterschiedliche Praktiken von Studierenden im Umgang mit digitalen Technologien, was bestätigt, dass es die „digital natives“ nicht gibt. Die explorativen Ergebnisse legen nahe, dass Studierende vielmehr unterschiedliche Hilfestellungen in Bezug auf die Digitalisierung des Studiums benötigen, die in der Lehrplanung und -pädagogik berücksichtigt werden müssten.

\section{Literatur}

Allert, Heidrun/Asmussen, Michael/Richter, Christoph (2018): Formen von Subjektivierung und Unbestimmtheit im Umgang mit datengetriebenen Lerntechnologien - eine praxistheoretische Position. In: Zeitschrift für Erziehungswissenschaft 21, H. 1, S. 142-158.

Becker, Rolf/Haunberger, Sigrid/Schubert, Frank (2010): Studienfachwahl als Spezialfall der Ausbildungsentscheidung und Berufswahl. In: Zeitschrift für ArbeitsmarktForschung 42, H. 4, S. 292-310. 
Bond, Melissa/Marín, Victoria I./Dolch, Carina/Bedenlier, Svenja/Zawacki-Richter, Olaf (2018): Digital transformation in German higher education: student and teacher perceptions and usage of digital media. In: International Journal of Educational Technology in Higher Education 15, H. 1.

Bourdieu, Pierre (1976): Entwurf einer Theorie der Praxis: auf der ethnologischen Grundlage der kabylischen Gesellschaft. Frankfurt am Main: Suhrkamp.

Bourdieu, Pierre (1987a): Die feinen Unterschiede: Kritik der gesellschaftlichen Urteilskraft. Frankfurt am Main: Suhrkamp.

Bourdieu, Pierre (1987b): Sozialer Sinn: Kritik der theoretischen Vernunft. Frankfurt am Main: Suhrkamp.

Bourdieu, Pierre/Passeron, Jean-Claude (1971): Die Illusion der Chancengleichheit: Untersuchung zur Soziologie des Bildungswesens am Beispiel Frankreichs. Stuttgart: Klett.

Bremer, Helmut/Teiwes-Kügler, Christel (2013): Habitusanalyse als Habitus-Hermeneutik. In: ZQF - Zeitschrift für Qualitative Forschung 14, H. 2, S. 199-219.

Carstensen, Tanja (2017): Digitalisierung als eigensinnige soziale Praxis. Arbeit 26, H. 1, S. 87-110.

Dauner-Lieb, Barbara/Kaulbach, Ann-Marie (2018): Editorial: Fünf Thesen zur Digitalisierung des Jurastudiums. In ebd. (Hrsg.): E-Learning im Jurastudium. Baden-Baden: Nomos, S. 712.

Deimann, Markus (preprint): Hochschulbildung und Digitalisierung - Entwicklungslinien und Trends für die 2020er Jahre. www.researchgate.net/publication/341152163_Hochschulbildung_und_Digitalisierung_-_Entwicklungslinien_und_Trends_fur_die_2020er_Jahre_1_PRE-PRINT_fur_den_Sammelband_Digitalisierung_in_Studium_und_Lehre_gemeinsam_gestalten (Abfrage: 08.05.2020).

Friese, Heidrun (2019): Digitalisierte Gesellschaften. In: ebd./Rebane, Gala/Nolden, Marcus/Schreiter, Miriam (Hrsg.): Handbuch Soziale Praktiken und Digitale Alltagswelten, Wiesbaden: Springer Fachmedien, S. 1-16.

Getto, Barbara/Hintze, Patrick/Kerres, Michael (2018): (Wie) Kann Digitalisierung zur Hochschulentwicklung beitragen? In: ebd (Hrsg.): Digitalisierung und Hochschulentwicklung. Proceedings zur 26. Tagung der Gesellschaft für Medien in der Wissenschaft e.V. Münster; New York: Waxmann, S. 13-25.

Helbig, Christian (2017): Die Mediatisierung professionellen Handelns. Zur Notwendigkeit von Handlungskompetenzen im Kontext digitaler Medien in der Sozialen Arbeit. In: MedienPädagogik: Zeitschrift für Theorie und Praxis der Medienbildung 27, S. 133-52. 
Houben, Daniel/Prietl, Bianca (Hrsg.) (2018): Datengesellschaft: Einsichten in die Datafizierung des Sozialen. Bielefeld: transcript Verlag.

Ignatow, Gabe/Robinson, Laura (2017): Pierre Bourdieu: theorizing the digital. Information, Communication \& Society 20, H. 7, S. 950-966.

Lange-Vester, Andrea/Teiwes-Kügler, Christel (2013): Das Konzept der Habitushermeneutik in der Milieuforschung. In: Lenger, Alexander/Schneickert Christian/Schumacher, Florian (Hrsg.): Pierre Bourdieus Konzeption des Habitus, Wiesbaden: Springer Fachmedien, S. 149-74.

Leger, Matthias/Panzitta, Susanne/Tiede, Maria (2018): Daten-Teilen? Digitale Selbstvermessung aus praxeologischer Perspektive. In: Houben, Daniel/Prietl, Bianca (Hrsg.): Datengesellschaft: Einsichten in die Datafizierung des Sozialen. Bielefeld: transcript. S. 35-59.

Lenger, Alexander/Schneickert Christian/Schumacher, Florian (2013): Pierre Bourdieus Konzeption des Habitus. In: ebd. (Hrsg.) Pierre Bourdieus Konzeption des Habitus: Grundlagen, Zugänge, Forschungsperspektiven. Wiesbaden: Springer Fachmedien, S. 11-41

Maiwald, Kai-Olaf (2003): Stellen Interviews eine geeignete Datenbasis für die Analyse beruflicher Praxis dar? In: sozialer sinn 4, H. 1, S. 151-180.

Meister, Dorothee M./Meise, Bianca (2010): Emergenz neuer Lernkulturen - Bildungsaneignungsperspektiven im Web 2.0. In: Herzig, Bardo/Meister, Dorothee M./Moser, Heinz/Niesyto, Horst (Hrsg.): Jahrbuch Medienpädagogik 8: Medienkompetenz und Web 2.0. Wiesbaden: VS Verlag für Sozialwissenschaften, S. 183-199.

Pensel, Sabrina/Hofhues, Sandra (2018): Digitale Lerninfrastrukturen an Hochschulen. Systematisches Review zu den Rahmenbedingungen für das Lehren und Lernen mit Medien an deutschen Hochschulen. omp.ub.rub.de/index.php/RUB/catalog/book/104 (Abfrage: 08.05.2020).

Persike, Malte/Friedrich, Julius-David (2016): Lernen mit digitalen Medien aus Studierendenperspektive. Sonderauswertung aus dem CHE Hochschulranking für die deutschen Hochschulen. Berlin: Stifterverband für die Deutsche Wissenschaft.

Robinson, Laura/Cotten, Shelia R./Ono, Hiroshi/Quan-Haase, Anabel/Mesch, Gustavo/Chen, Wenhong/Schulz, Jeremy/Hale, Timothy M./Stern, Michael J. (2015): Digital inequalities and why they matter. In: Information, Communication \& Society 18, H. 5, S. 569-582.

Schäfer, Hilmar (2016a): Praxis als Wiederholung. Das Denken der Iterabilität und seine Konsequenzen für die Methodologie praxeologischer Forschung. In: ebd. (Hrsg.): Praxistheorie: ein soziologisches Forschungsprogramm Bielefeld: transcript. 
Schäfer, Hilmar (Hrsg.) (2016b): Praxistheorie: ein soziologisches Forschungsprogramm. Bielefeld: transcript.

Schmid, Johannes C. P./Veits, Andreas/Vorrath, Wiebke (Hrsg.) (2018): Praktiken medialer Transformationen: Übersetzungen in und aus dem digitalen Raum, Bielefeld: transcript.

Schmidt, Robert (2012): Soziologie der Praktiken: Konzeptionelle Studien und empirische Analysen. Frankfurt am Main: Suhrkamp.

Schmitt, Lars (2010): Bestellt und nicht abgeholt. Wiesbaden: VS Verlag für Sozialwissenschaften.

Schütze, Fritz (1977): Die Technik des narrativen Interviews in Interaktionsfeldstudien: dargestellt an einem Projekt zur Erforschung von kommunalen Machtstrukturen. Bielefeld: Universitätsverlag.

Spangenberg, Heike/Beuße, Mareike/Heine, Christoph (2011): Nachschulische Werdegänge des Studienberechtigtenjahrgangs 2006. Dritte Befragung der Studienberechtigten Schulabgänger/innen 20063 1⁄2 Jahre nach Schulabschluss im Zeitvergleich. HIS:Forum Hochschule 18. Hannover: HIS Hochschul-Informations-System GmbH.

Steffens, Yannic/Schmitt, Inga Lotta/Aßmann, Sandra (2018): Mediennutzung Studierender: Über den Umgang mit Medien in hochschulischen Kontexten. Systematisches Review nationaler und internationaler Studien zur Mediennutzung Studierender. omp.ub.rub.de/index.php/RUB/catalog/book/106 (Abfrage: 05.04.2019).

Steinhardt, Isabel (2018): Open Science-Forschung und qualitative Methoden - fünf Ebenen der Reflexion. In: MedienPädagogik: Zeitschrift für Theorie und Praxis der Medienbildung 32, S. 122-138.

Steinhardt, Isabel (2020): Learning Open Science by doing Open Science. A reflection of a qualitative research project-based seminar. In: Education for Information.

Steinhardt, Isabel/İkiz-Akınc1, Dilek (2020): Digitale Bildungspraktiken von Studierenden (DEPS). Daten- und Methodenbericht zur Studie DEPS 2018. Version 1.0.0. Kassel/Hannover.

Teiwes-Kügler, Christel/Lange-Vester, Andrea (2018): Das Konzept der Habitus-Hermeneutik in der typenbildenden Milieuforschung. In: Müller, Stella/Zimmermann, Jens (Hrsg.): Milieu - Revisited: Forschungsstrategien der qualitativen Milieuanalyse, Wiesbaden: Springer Fachmedien, S. 113-155.

Wannemacher, Klaus (2016): Digitale Lernszenarien im Hochschulbereich. Arbeitspapier 15 Berlin: hochschulforum digitalisierung. 
Zillien, Nicole. 2013. Wissenskluftforschung. In: Schweiger, Wolfgang/Fahr, Andreas: Handbuch Medienwirkungsforschung, Wiesbaden: Springer Fachmedien, S. 495-512.

Zillien, Nicole/Fröhlich, Gerrit/Kofahl, Daniel (2016): Ernährungsbezogene Selbstvermessung. Von der Diätetik bis zum Diet-Tracking. In: Duttweiler, Stefanie/Gugutzer, Roland/Passoth, Jan-Hendrik/Strübing, Jörg: Leben nach Zahlen: Self-Tracking als Optimierungsprojekt? Bielefeld: transcript, S. 123-140.

Zillien, Nicole/Marr, Mirco (2013): The Digital Divide in Europe. In: Ragnedda, Massimo/ Muschert, Glenn W.: The Digital Divide: The Internet and Social Inequality in International Perspective. Routledge. 